\title{
Graphene Oxide and Polyethyleneimine Cooperative Construct Ionic Imprinted Cellulose Nanocrystals Aerogel for Selective Adsorption of Dy(III)
}

Xudong Zheng ( $\sim$ Zhengks@outlook.com )

Changzhou University https://orcid.org/0000-0003-2688-4855

\section{Wen Sun}

Changzhou University

Ang Li

Changzhou University

\section{Bin Wang}

Changzhou University

\section{Rong Jiang}

Changzhou University

\section{Zhiqiang Song}

Changzhou University

\section{Yuzhe Zhang}

Changzhou University

\section{Zhongyu Li}

Changzhou University

\section{Research Article}

Keywords: Cellulose nanocrystals, Aerogel, Graphene oxide, Dysprosium, Adsorption

Posted Date: June 21st, 2021

DOl: https://doi.org/10.21203/rs.3.rs-586534/v1

License: (c) (1) This work is licensed under a Creative Commons Attribution 4.0 International License. Read Full License 
1 Graphene oxide and polyethyleneimine cooperative construct ionic

2 imprinted cellulose nanocrystals aerogel for selective adsorption of

3

4

5

6

7

8

9

10

\section{Dy(III)}

Xudong Zheng ${ }^{\mathrm{a}^{*}}$, Wen Sun ${ }^{\mathrm{a}}$, Ang Li ${ }^{\mathrm{a}}$, Bin Wang ${ }^{\mathrm{a}}$, Rong Jiang ${ }^{\mathrm{a}}$, Zhiqiang Song ${ }^{\mathrm{a}}$, Yuzhe

Zhang ${ }^{\mathrm{a}}$, Zhongyu Li ${ }^{\mathrm{a}, \mathrm{b}, \mathrm{c}}$

a School of Environmental and Safety Engineering, Changzhou University, Changzhou, 213164, PR China

${ }^{\mathrm{b}}$ Jiangsu Key Laboratory of Advanced Catalytic Materials and Technology, School of Petrochemical Engineering, Changzhou University, Changzhou, 213164, PR China

${ }^{\mathrm{c}}$ Advanced Catalysis and Green Manufacturing Collaborative Innovation Center, Changzhou University, Changzhou, 213164, PR China

* Corresponding author

Postal address: Mingxing Building, Science and Education City, Wujin District, Changzhou, Jiangsu, China

Mail addresses: zhengks@outlook.com 


\section{Abstract}

Because of dysprosium's unique physical and chemical properties and limited supply, the price of rare earth dysprosium has been high in recent years. Therefore, the study of the method of high efficiency selective separation of dysprosium has the double value of scientific research and practical economy. In this paper, we used periodic cellulose nanocrystals as the basic structure, polyethylenimine and graphene oxide were introduced, combined with imprinting technology, to construct porous imprinted aerogel and use it for selective adsorption of Dy(III). The physical and chemical properties were characterized by SEM, TEM, FT-IR and TGA. It was proved that both polyethylenimine and graphene oxide were crosslinked effectively with cellulose nanocrystals. Adsorption experiments showed that the composite imprinted aerogel could selectively adsorb dysprosium effectively, and the maximum adsorption capacity for Dy(III) was $39.027 \mathrm{mg} \mathrm{g}^{-1}$. The reproducibility experiment showed that aerogel had good regeneration ability. In conclusion, cellulose nanocrystals aerogel, which is environmentally friendly, efficient and repeatable, is expected to provide a new direction for the recovery of rare earth elements.

Keywords: Cellulose nanocrystals; Aerogel; Graphene oxide; Dysprosium; Adsorption 


\section{Introduction}

Rare earth elements, known as "industrial vitamins", are widely used in catalysts, permanent magnets, glassmaking, lighting and other fields(Huang and Zhu 2019; Liang et al. 2018; Ni'am et al. 2020; Zhao et al. 2019). Dysprosium (Dy) has always been one of the most important rare earth elements because of its irreplaceable role in optics and permanent magnets(Bisaka et al. 2017; Fujiwara et al. 2016; Liang et al. 2018; Zheng et al. 2020). Moreover, China has attached great importance to the rational development of rare earth resources in recent years. The price of rare earth has changed from "soil" to "rare". Therefore, it is of great significance for sustainable development to explore an economical and green separation and recovery technologies for dysprosium(Kaneko et al. 2019; Prodius et al. 2020).

Since the middle of the 20th century, many scholars began to pay attention to the processing and recycling of dysprosium ions(Balaram 2019; Habib 2019). In various methods of recovering dysprosium ions, adsorption is an effective alternative because of its simplicity and cost-effectiveness(Kegl et al. 2019). Alcaraz(Alcaraz et al. 2019) synthesized two kinds of activated carbons from waste coffee grounds to adsorb and remove dysprosium ions in aqueous solution. Kaneko(Kaneko et al. 2019) synthesized MPS materials with high selective adsorption properties for dysprosium ions, which provided a new idea for the separation and recovery of dysprosium ions. On the basis of previous studies, we found that the selective adsorption of dysprosium is still a difficult problem because the physical and chemical properties of rare earth elements are directly similar. In addition, the cost of adsorption materials still restricts the 
industrial application of adsorption process. Therefore, researches on green, low-cost, efficient adsorption materials for selective adsorption of Dy is one of current researching hot directions.

Cellulose nanocrystals (CNCs) are considered as a kind of promising aerogel material due to their green, natural nature and low price(Almeida et al. 2018; Du et al. 2019; Zhang and Zhang 2020). CNCs prepared by sulfuric acid hydrolysis usually have a diameter of 5-20 nanometers and a length of 10-hundreds of nanometers, and a large number of hydroxyl groups exist on their surfaces(George and S N 2015; Kontturi et al. 2018). Since CNCs can form chiral nematic structures, aerogels made by CNCs have a natural ordered pore structure( $\mathrm{Xu}$ et al. 2018), which is conducive to the occurrence of adsorption process(Cao et al. 2020; Kim et al. 2014; Sato et al. 2004). However, the similar properties of other rare earth elements to Dy(III) limit the separation of Dy(III) as a single element, so we introduced imprinting technology(Fang et al. 2021; Zhang et al. 2021) to fix the recognition sites on the surface of CNCs, so as to carry out more effective selective adsorption.

Aerogel has high specific surface area, high porosity and high adsorption capacity, and is one of the materials with the lowest density at present(Du et al. 2013; Salimian et al. 2018), which has been applied in multiple scenarios such as heat insulation materials, storage electrical parts or oil adsorbents, and is a very promising adsorbent material. However, because the colloidal particles in the sol are usually randomly distributed, rapid drying of the gel to aerogel usually produces disordered networks(Long et al. 2018). The technology for preparing ordered aerogels is not well 
developed. To solve the problem, we introduced ordered $\mathrm{CNCs}$ and printed it into aerogels(Xu et al. 2018).

At present, there is not much research on imprinted aerogels, so we aim to introduce imprinting into aerogels and modify them so that the aerogel can adsorb Dy(III) selectively. The branching end of polyethyleneimine (PEI) is rich in primary amine groups, which can easily form crosslinking points with other functional groups, so it is often used in the surface modification of nanomaterials(Zhao et al. 2017). Graphene oxide (GO) has lots of carboxyl, hydroxyl and epoxy groups on its surface, which has the characteristics of high specific surface area, high strength, good chemical stability and so on(Ashour et al. 2017). As an adsorbent, GO has a high adsorption capacity. Using cellulose nanocrystals as the framework, we prepared a green imprinted GO-CNCs-PEI aerogel (IGCPA) with high imprinted adsorption capacity and high selectivity by using ion imprinting technology(Fu et al. 2015) and using GO and PEI as bifunctional monomers to conduct selective adsorption separation of Dy(III)(Jiang et al. 2020; Stepanova et al. 2019), and the cross-linking of GO can also improve the structural strength of the aerogel.

\section{Experimental section}

\subsection{Materials}

Skimmed cotton was bought at a drugstore. Hydrochloric acid (HCl, 37\%), Sulfuric acid $\left(\mathrm{H}_{2} \mathrm{SO}_{4}, 98 \%\right)$, Nitric acid $\left(\mathrm{HNO}_{3}, 68 \%\right)$, Glacial acetic acid $\left(\mathrm{CH}_{3} \mathrm{COOH}\right.$, 99.5\%), Sodium hydroxide ( $\mathrm{NaOH}, 96 \%)$, 2,2,6,6-Tetramethyl-1-piperidinyloxy (TEMPO, 97\%), Ethanol $\left(\mathrm{C}_{2} \mathrm{H}_{5} \mathrm{OH}, 99.7 \%\right)$, Sodium bromide $(\mathrm{NaBr}, 99 \%)$ and Sodium hypochlorite $(\mathrm{NaClO}$, Active chlorine $\geq 5.2 \%$ ) were purchased from Sigma Aldrich. 
Dysprosium oxide (TREO $\geq 99.9 \%$ ) was purchased from Shanghai Sinian Metal Materials Co., Ltd. Polyethyleneimine (PEI, 50\%), 1-(3-Dimethylaminopropyl)-3ethylcarbodiimide hydro (EDC, 98\%) and N-Hydroxy succinimide (NHS, 98\%) were bought from Aladdin Biochemical Technology Co., Ltd. Graphene oxide solutions (GO, $10 \mathrm{mg} \mathrm{mL}^{-1}$ ) were purchased from Nanjing Xianfeng Nanomaterials Technology Co., Ltd. The dysprosium nitrate $\left(\mathrm{Dy}\left(\mathrm{NO}_{3}\right)_{3}\right)$ used in this study was prepared by dissolving dysprosium oxide with excessive concentrated nitric acid and reheating. The water used in this experiment was distilled water, and all reagents were not further purified unless otherwise specified.

\subsection{Instruments}

Scanning electron microscope (SEM, JEOL, Japan) was used to observe the surface morphology of the aerogel. The microstructure of the sample was observed using a $300 \mathrm{kV}$ transmission electron microscope (TEM, JEOL 2100, Japan). The samples were tested by a Fourier transform infrared spectrophotometer (FT-IR, IS50, United States). Automatic specific surface and porosity analyzer (BET, ASAP 2460, USA) was used to analyze the specific surface area $\left(S_{\mathrm{BET}}\right)$ of aerogels. Under $\mathrm{N}_{2}$ atmosphere, the aerogels were subjected to thermogravimetric analysis using a thermogravimetric analyzer (Q600-TGA/DSC, United States). The concentration of Dy(III) was measured by Inductively coupled plasma atomic emission spectrometer (ICP-OES, Vista-AX, United States).

\subsection{Preparation of Samples}

The synthesis of CNCs suspension: Concentrated sulfuric acid (150 mL) was added to water $(150 \mathrm{~mL})$, stirred evenly and cooled to room temperature. Skimmed cotton $(20 \mathrm{~g})$ was put into the mixture, stirred it for 2 hours at room temperature, then 
poured it into ice water $(3000 \mathrm{~mL})$, and let it stand for 15 hours for precipitation. The suspension of the lower phase was centrifuged and purified by dialysis until the $\mathrm{pH}$ value of the suspension was greater than 2.4 .

The synthesis of TEMPO-CNCs suspension: After the CNCs solution $(100 \mathrm{~mL}$, 4wt\%) was evenly dispersed in $200 \mathrm{~mL}$ water, TEMPO $(10 \mathrm{mg})$ and $\mathrm{NaBr}(100 \mathrm{mg})$ were added to soak in the solution for 1 hour. Added $\mathrm{NaClO}$ solution $(40 \mathrm{~mL})$ drop by drop, maintain $\mathrm{pH}$ value of 10.5 with $\mathrm{HCl}(0.1 \mathrm{M})$ and $\mathrm{NaOH}(0.1 \mathrm{M})$, and stirred for 4 hours. Finally, ethanol $(4 \mathrm{~mL})$ was added to stop the oxidation reaction. $\mathrm{HCl}(0.1 \mathrm{M})$ was used to adjust the solution to $\mathrm{pH}=7.0$. After standing overnight, dialysate was carried out and TEMPO-CNCs suspension was obtained.

The synthesis of PEI-CNCs aerogel: PEI (1000 mg, 50\%) was dispersed in the TEMPO-CNCs suspension $(50 \mathrm{~mL})$, followed by ultrasonic for 30 minutes, then 1-(3Dimethylaminopropyl)-3-ethylcarbodiimide hydro (EDC, $250 \mathrm{mg}$ ) and N-hydroxy succinimide (NHS, $250 \mathrm{mg}$ ) were added into the mixed solution, stirred at room temperature for 24 hours, and dialyzed to obtain a yellow suspension. After freeze drying, PEI-CNCs aerogel was prepared.

The synthesis of GO-CNCs-PEI aerogel (GCPA): Graphene oxide (GO, $40 \mathrm{mg}$ ) were added to PEI-CNCs suspension $(20 \mathrm{~mL})$, the $\mathrm{pH}$ value was adjusted to 5.0 with $\mathrm{HCl}(0.1 \mathrm{M})$, and the solution was stirred for 3 hours. GCPA was obtained by freezedrying after dialysis purification.

The synthesis of IGCPA: GO (40 mg) and Dy( $\left.\mathrm{NO}_{3}\right)_{3}(10 \mathrm{mg})$ were added to PEICNCs suspension $(20 \mathrm{~mL})$, the $\mathrm{pH}$ value was adjusted to 5.0 by $\mathrm{HCl}(0.1 \mathrm{M})$ and stirred 
151

152

153

154

155

156

for 3 hours. Freeze drying after dialysis purification. Transferred the aerogel in a eluent (the volume ratio of glacial acetic acid to water is 1:9) for 48 hours to remove Dy(III). Finally, the IGCPA was dried at room temperature after being rinsed three times with ultra-pure water. The preparation process and the possible adsorption mechanism of aerogel are shown in Figure 1 and Figure 2.

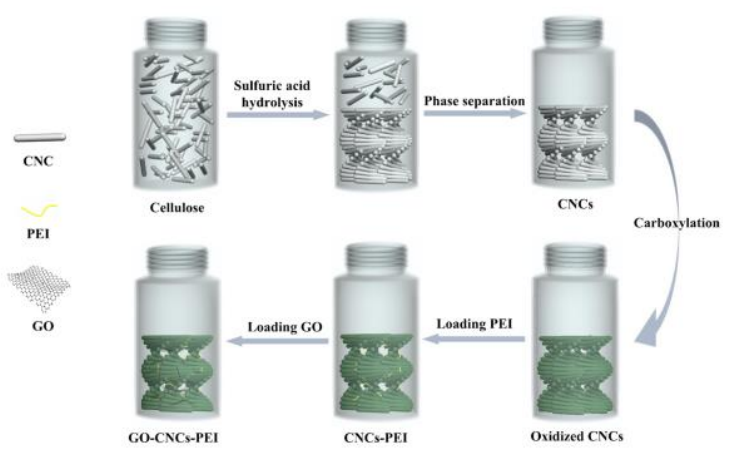

Figure 1 Synthesis of cellulose nanocrystals aerogel
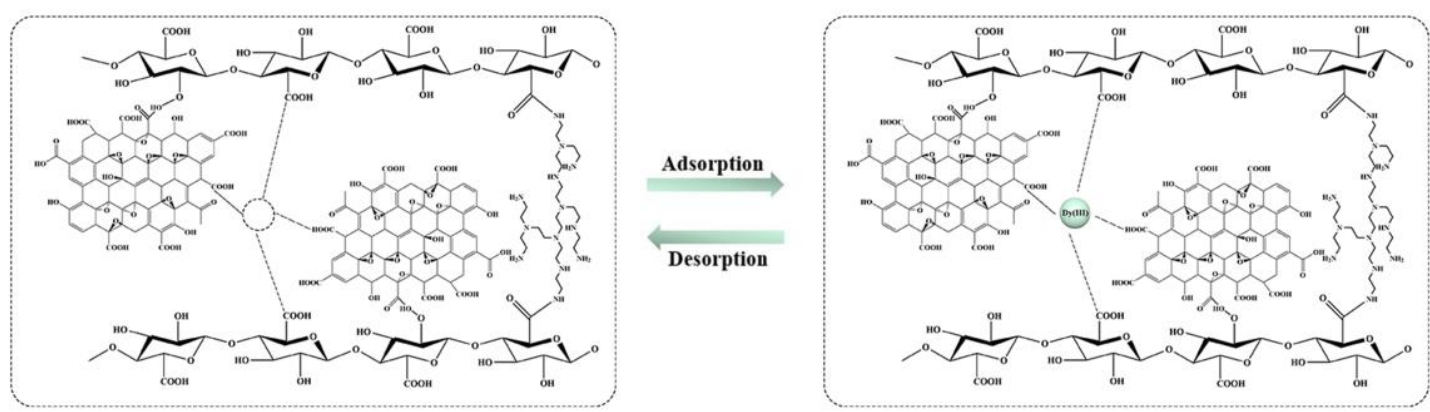

Figure 2 Possible adsorption mechanism for Dy(III) onto IGCPA.

\subsection{Adsorption experiment}

Effect of pH on adsorption: $\mathrm{Dy}\left(\mathrm{NO}_{3}\right)_{3}$ was used to prepare dysprosium ion original solution with a concentration of $50 \mathrm{mg} \mathrm{L}^{-1}$. We took out the original solution and adjusted the $\mathrm{pH}$ value to $2.0,3.0,4.0,5.0,6.0$ and 7.0 with $0.1 \mathrm{M} \mathrm{HCl}$, respectively. Then we took the solution and placed them in centrifuge tubes. Three aerogels were then placed in a mixture of solutions in a centrifuge tube and allowed to sit for 24 hours. 
After adsorption was completed, the mixture was centrifuged (10000 $\mathrm{r} \mathrm{min}^{-1}, 15 \mathrm{~min}$ ) and the absorbents was removed using a $0.22 \mu \mathrm{m}$ filter. The concentration of Dy(III) in the mixed solution was measured by the ICP-OES at wavelength of $353.17 \mathrm{~nm}$. The adsorption capacities $Q_{\mathrm{t}}\left(\mathrm{mg} \mathrm{g}^{-1}\right)$ were calculated by the following formula(Mahdi et al. 2018):

$$
Q_{\mathrm{t}}=\frac{V\left(C_{0}-C_{\mathrm{t}}\right)}{m}
$$

Where $C_{0}$ and $C_{\mathrm{t}}\left(\mathrm{mg} \mathrm{L}^{-1}\right)$ are initial concentration and residual concentration of Dy(III) in mixed solution, respectively. $V(\mathrm{~L})$ is the volume of Dy(III) stock solution, and $m(\mathrm{~g})$ is the mass of aerogel.

Adsorption dynamics: Three Dy(III) original solution $(10 \mathrm{~mL})$ were prepared, $\mathrm{pH}$ was adjusted to 5.0, and placed in centrifuge tubes. Three kinds of aerogels $(10 \mathrm{mg})$ were put into the tubes. The concentrations of dysprosium ions in mixed solution were determined at different contact times ( 5 minutes to 24 hours). We used the q pseudofirst-order kinetic model (PFOKM) and pseudo-second-order kinetic model (PSOKM) respectively to fit the adsorption kinetic data, and then analyzed the adsorption mechanism.

The pseudo-first-order kinetic model (PFOKM) and pseudo-second-order kinetic model (PSOKM) were used to fit the dynamics data and explore adsorption rate constants and adsorption mechanisms(Wang et al. 2017).

$$
\begin{gathered}
Q_{\mathrm{t}}=Q_{\mathrm{e}}-Q_{\mathrm{e}} \mathrm{e}^{-k_{1} t} \\
Q_{\mathrm{t}}=\frac{k_{2} Q_{\mathrm{e}}^{2} t}{1+k_{2} Q_{\mathrm{e}} t}
\end{gathered}
$$

Where, $Q_{\mathrm{t}}\left(\mathrm{mg} \mathrm{g}^{-1}\right)$ and $Q_{\mathrm{e}}\left(\mathrm{mg} \mathrm{g}^{-1}\right)$ are the adsorption amount of aerogels at time 
$t$ and equilibrium, respectively. $k_{1}\left(\mathrm{~min}^{-1}\right)$ and $k_{2}\left(\mathrm{~g} \mathrm{mg}^{-1} \mathrm{~min}^{-1}\right)$ represent the rate constants of PFOKM and PSOKM, respectively.

Adsorption isotherms: Dy(III) solutions with concentrations of 25, 50, 100, 150 and $200 \mathrm{mg} \mathrm{L}^{-1}$ were taken, adjusted to $\mathrm{pH}$ 5.0, and placed in centrifuge tubes. Three different kinds of aerogels $(10 \mathrm{mg})$ were put into the mixed solutions. The adsorption of Dy(III) by aerogels at different values of initial concentrations was determined 24 hours after the tubes was placed. Langmuir (Eq. (4)) and Freundlich (Eq. (5)) models were used to fit the experimental equilibrium data(Zhu et al. 2018).

$$
\begin{gathered}
Q_{\mathrm{e}}=\frac{K_{\mathrm{L}} Q_{\mathrm{m}} C_{\mathrm{e}}}{1+K_{\mathrm{L}} C_{\mathrm{e}}} \\
Q_{\mathrm{e}}=K_{\mathrm{F}} C_{\mathrm{e}}^{1 / n}
\end{gathered}
$$

$C_{\mathrm{e}}\left(\mathrm{mg} \mathrm{L}^{-1}\right)$ is the concentration of Dy(III) in solution at equilibrium, $Q_{\mathrm{m}}\left(\mathrm{mg} \mathrm{g}^{-1}\right)$ is the maximum adsorption capacity of aerogels for Dy(III). $K_{\mathrm{L}}\left(\mathrm{L} \mathrm{mg}^{-1}\right)$ is the Langmuir parameter denoted the energy of adsorption and affinity of binding sites. $K_{\mathrm{F}}\left(\mathrm{mg} \mathrm{g}^{-1}\right)$ is the Freundlich sorbent adsorption strength, while $1 / n$ is the heterogeneity factors.

Adsorption thermodynamics: We took stock solutions (50 $\left.\mathrm{mg} \mathrm{L}^{-1}\right)$, adjusted the $\mathrm{pH}$ to 5.0, and put them into the centrifuge tubes. Three different aerogels were then put into the mixed solutions. The residual Dy(III) concentration in the solution was determined 24 hours after the tubes was placed at $25^{\circ} \mathrm{C}$. Then the above temperature was changed to $35^{\circ} \mathrm{C}$ and $45^{\circ} \mathrm{C}$ respectively, and the experiment was repeated twice to study the adsorption effect at different temperatures. The values of Gibbs energy $\left(\Delta G^{\circ}\right)$ are calculated by equation Eq. (6)(Zhang et al. 2019):

$$
\Delta G^{\circ}=-R T \ln K^{\circ}
$$

Where thermodynamic equilibrium constant $K^{\circ}$ is calculated by plotting the 
function of $\ln \left(C_{\mathrm{s}} / C_{\mathrm{e}}\right)$ versus $C_{\mathrm{s}}$. Wherein, $C_{\mathrm{s}}\left(\mathrm{mmol} \mathrm{g}^{-1}\right)$ is the adsorption amount per gram of aerogels, and $C_{\mathrm{e}}$ is the concentration of Dy(III) in the mixed solution at adsorption equilibrium. $R\left(8.314 \mathrm{~J} \mathrm{~mol}^{-1} \mathrm{~K}^{-1}\right)$ is the universal gas constant, and $T$ is the given temperature. Eventually, entropy $\left(\Delta S^{\circ}\right)$ and the enthalpy $\left(\Delta H^{\circ}\right)$ values are obtained from van't Hoff equation Eq. (7)(Zheng et al. 2020):

$$
\ln K^{\circ}=\frac{\Delta S}{R}-\frac{\Delta H}{R T}
$$

Selective tests: $10 \mathrm{mg}$ aerogels were immersed in a coexisting system (Dy(III), $\mathrm{Nd}(\mathrm{III})$ and $\operatorname{Pr}(\mathrm{III}))$. The initial concentration of each cation was $50 \mathrm{mg} \mathrm{\textrm {L } ^ { - 1 }}$. The concentration in the mixed solution was measured 24 hours after the tubes was placed at $25^{\circ} \mathrm{C}$. The selectivity of aerogels was evaluated by $K_{\mathrm{d}}\left(\mathrm{mL} \mathrm{g}^{-1}\right)$. Relationships are listed as follow (Eq. (8))(Sun et al. 2017):

$$
K_{\mathrm{d}}=\frac{V\left(C_{0}-C_{\mathrm{f}}\right)}{m C_{\mathrm{f}}}
$$

Where, $C_{0}$ and $C_{\mathrm{f}}$ are initial and final concentration of each rare earth ion, respectively. The $C_{0}$ of each cation is $50 \mathrm{mg} \mathrm{L}^{-1}$.

Reusability tests: After each adsorption process, the aerogels was separated from the solution by elution ( $10 \%$ glacial acetic acid solution) for 24 hours to remove the adsorbed Dy(III). These aerogels were then reapplied to the adsorption experiment. The whole adsorption-desorption experiment was repeated 5 times.

\section{Results and discussion}

\subsection{Aerogels characterization}

SEM analysis: The microstructure and surface morphology of composite aerogels were observed by scanning electron microscopy (SEM), as shown in Figure 3. Under 
the combined action of GO and PEI, the materials showed good porous structure. It can be seen that the material is composed of a large number of pores, which contributes to the ultra-light performance of the aerogels and also provides lots of adsorption sites for Dy(III) adsorption. The pores on the surface of PEI-CNCS aerogels, GCPA and IGCPA all have good periodicity, indicating that the composite aerogels can effectively replicate the structure of $\mathrm{CNCs}$, the subsequent modification and the elution of

231 imprinted ions have no effect on the structure of aerogels, which effectively maintain

232 the periodicity template of the materials. In addition, in Figure 3 (b) and Figure 3 (c),

233 PEI and GO are uniformly distributed on the porous network of CNCs, indicating that 234 the cross-linking between PEI and GO and CNCs is stable. The adhesion of GO to 235 CNCs not only added additional adsorption sites, but also prevented the agglomeration of CNCs to a certain extent, which contributed to the efficient adsorption of Dy(III) by

237 the adsorbent.
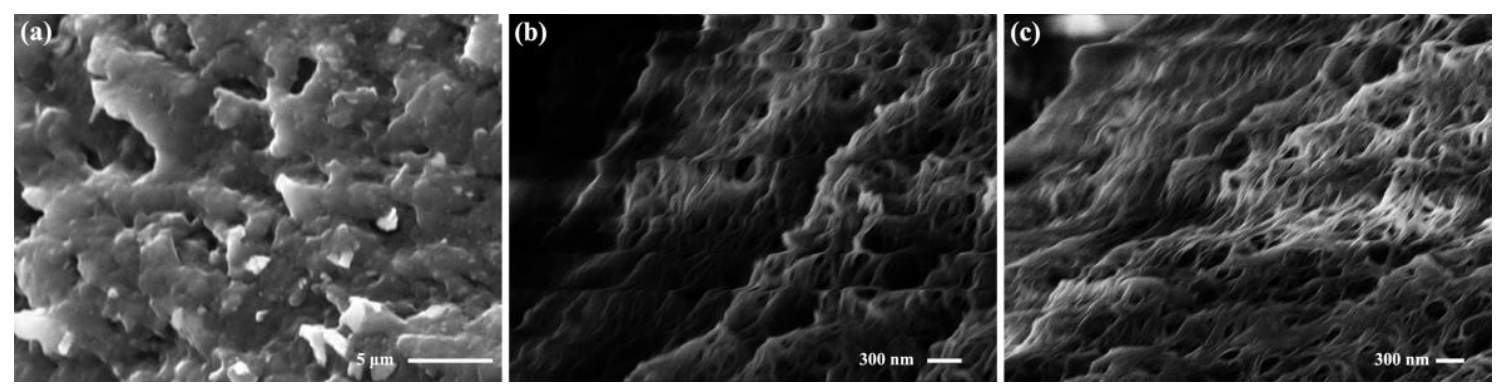

Figure 3 SEM images of PEI-CNCs aerogel (a), GCPA (b) and IGCPA(c).

TEM analysis: TEM images (Figure 4) shows that the three aerogels all exhibit uniformly arranged pore structures, which match the size in SEM images, confirming

242 the successful preservation of periodic structures. Compared with PEI-CNCs, GCPA 243 and IGCPA increased the flake morphology of GO, indicating that GO can successfully 
adhere to $\mathrm{CNCs}$ and maintain the original structure. Compared with non-imprinted aerogels, the eluted imprinted aerogels also have better pore structure and show good stability. All these are consistent with the SEM results mentioned above. In addition, the elemental composition of IGCPA was analyzed by EDS, as shown in Figure 4 (d). The elemental analysis results are consistent with the expected C, N, and O elements. The cross-linking reaction of PEI increased the intensity of $\mathrm{N}$ signal, and dysprosium

250 was not detected, which further confirmed that the cross-linking reaction was an effective method for preparing amine-rich porous aerogels.
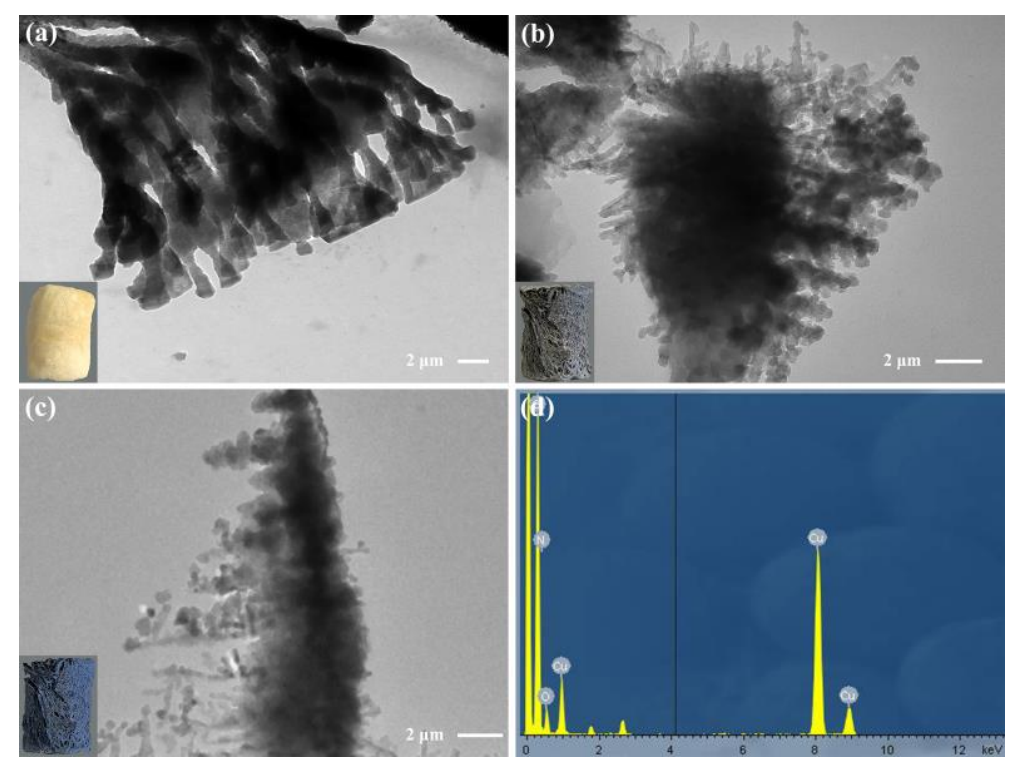

Figure 4 TEM images of PEI-CNCs aerogel (a), GCPA (b) and IGCPA (c), EDS element analysis of IGCPA (d).

Figure 5. It can be seen from the isotherm fitting curve that the three kinds of aerogels adsorption processes all belong to type IV. When the relative pressure is in the lowpressure region $\left(0.0-0.3 P / P_{0}\right)$, the upward curve is a monolayer adsorption process. 
260

261

262

As the pressure increases, the pore size of the aggregate expands, resulting in the subsequent phenomenon. The curves of adsorption return line adsorption and desorption formed are very steep, and the relative pressure of condensation and evaporation is in the middle. Therefore, the surface of IGCPA is a cylindrical hole with openings at both ends, which is consistent with the results of SEM images. In addition, the $S_{\mathrm{BET}}$ of PEI-CNC, GCPA and IGCPA were 4.175, 10.832 and $14.372 \mathrm{~m}^{2} \mathrm{~g}^{-1}$, respectively. As CNCs were further functionalized by PEI and GO, $S_{\mathrm{BET}}$ increased. This may be due to the new pores created by PEI and GO on the surface of CNCs, resulting in the decrease of pore size and the increase of $S_{\mathrm{BET}}$ of the materials. In addition, the specific surface area of composite aerogels is generally smaller, most likely due to the formation of large aggregates or aggregates during drying(Brinkmann et al. 2016; Peng et al. 2011; Zheng et al. 2020).

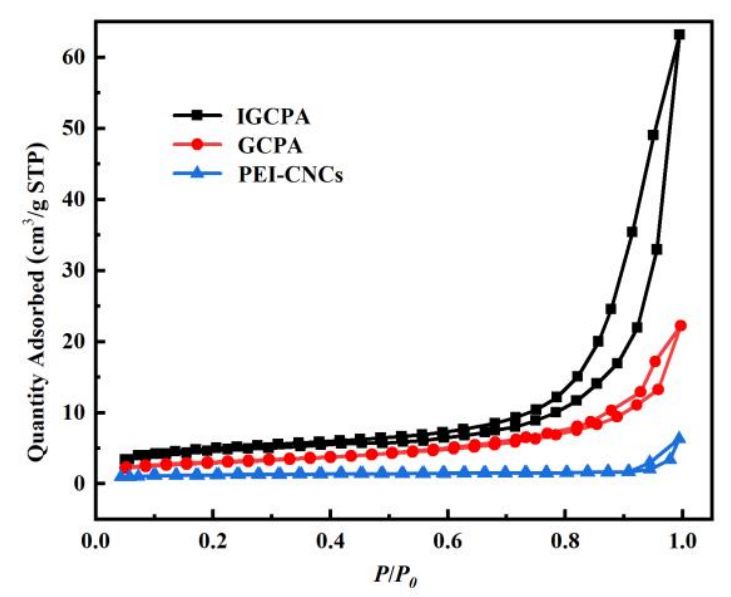

Figure $5 \mathrm{~N}_{2}$ adsorption-desorption isotherms of PEI-CNCs, GCPA and IGCPA.

FT-IR analysis: Infrared spectra were measured, and FT-IR spectra of TEMPOCNCs after freeze drying and three aerogels were shown in Figure 6. It is worth noting that the spectrum of TEMPO-CNCs has a strong peak at $1627 \mathrm{~cm}^{-1}$, which is a 
characteristic peak of the carbonyl of the carboxylic acid groups. There were three strong peaks in PEI-CNCs spectrum at 1644, 1567 and $1461 \mathrm{~cm}^{-1}$, indicating the characteristic peaks of amide bond and amino group. In addition, PEI-CNCs showed a strong spectral band in the range of $3100-3550 \mathrm{~cm}^{-1}$, which was due to the stretching vibration of $\mathrm{O}-\mathrm{H}$ and $\mathrm{N}-\mathrm{H}$ at $3100-3500 \mathrm{~cm}^{-1}$. Therefore, it can be proved that the amino group has been successfully introduced in the cross-linking process of PEI. The peaks of GCPA and IGCPA at $2904 \mathrm{~cm}^{-1}$ were observed to change, which was attributed to the stretching vibration of $-\mathrm{CH}_{2}$ due to defects in the graphite structure. It can be concluded that GO was successfully introduced into GCPA and IGCPA. In addition, the peaks of eluted IGCPA were consistent with that of GCPA, indicating that imprinted ions in IGCPA were completely washed and other groups remained stable, which proved that the modified material had good stability.

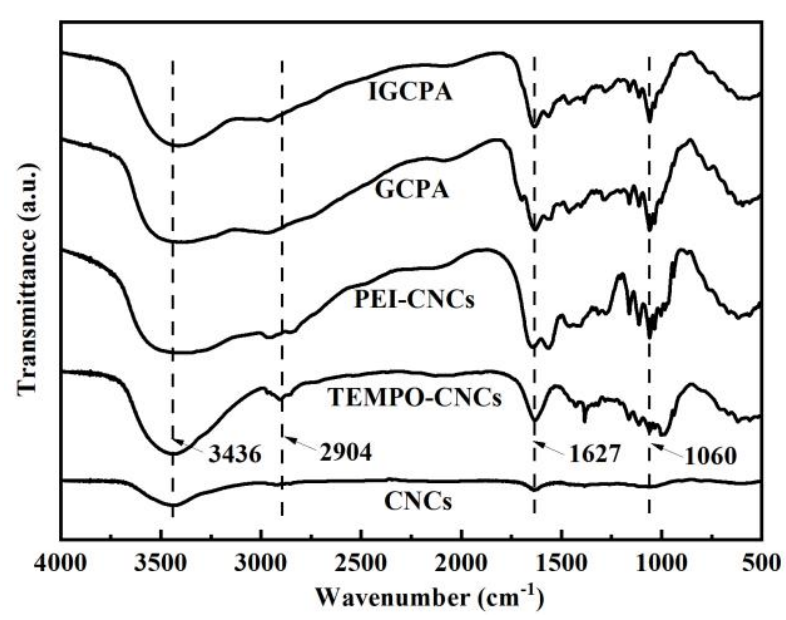

Figure 6 FT-IR spectra of aerogels.

TG/DTG analysis: The thermal stability of the material was investigated by increasing the temperature from $30^{\circ} \mathrm{C}$ to $800^{\circ} \mathrm{C}$ in $\mathrm{N}_{2}$ atmosphere. The thermogravimetric results are shown in Figure 7. When the temperature reaches $250^{\circ} \mathrm{C}$, 
decomposition is obvious and the mass decreases by $15-25 \%$, which is mainly due to water loss. The total mass loss between $250-400^{\circ} \mathrm{C}$ can be explained by the decomposition of the nanofiber network structure. Above $400^{\circ} \mathrm{C}$, entering the final carbonization stage, the mass loss gradually stops, the TGA curve flattens out, and the residue is mainly composed of carbon left by calcination. The above results show that the addition of GO, PEI and other materials improves the thermal stability of the materials. The total mass loss rate of IGCPA was less than that of GCPA, indicating that the imprinted aerogels had better thermal stability.
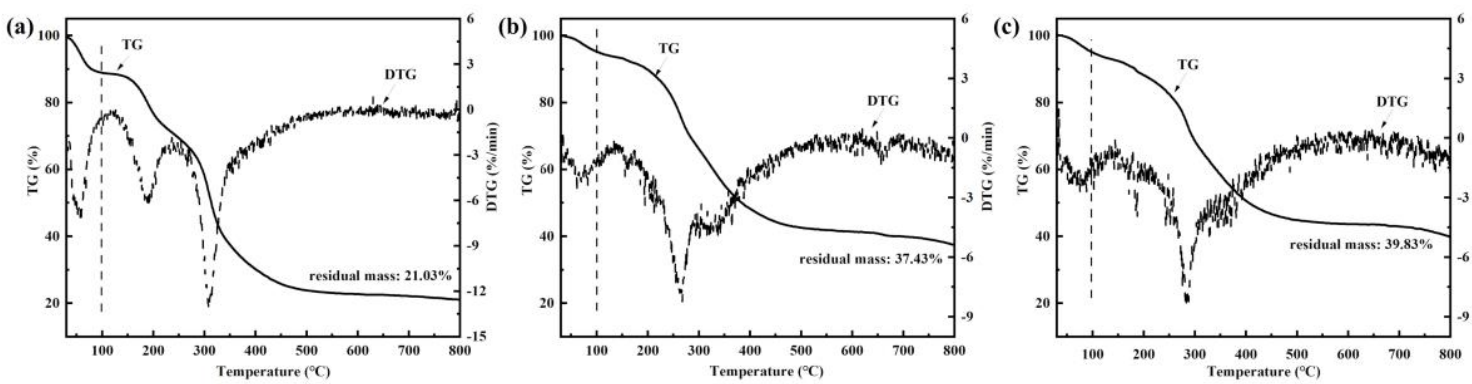

Figure 7 TGA curves of PEI-CNCs (a), GCPA (b) and IGCPA (c).

\subsection{Analysis of adsorption results}

Effect of pH on adsorption: The $\mathrm{pH}$ value affects the presence of cations in the solution and the surface charge of the adsorbent. In order to avoid the precipitation of dysprosium hydroxide under alkaline conditions, the adsorption properties in the $\mathrm{pH}$ range of 2.0-7.0 were studied. As can be seen from Figure 8, with the increase of $\mathrm{pH}$ value, the adsorption amount of each adsorbent gradually increases. The adsorption capacity increased rapidly in the $\mathrm{pH} 1.0-5.0$ range. When the $\mathrm{pH}$ value was higher than 5.0, the adsorption capacity gradually stabilized, which could be attributed to the low degree of dissociation of the -COOH group. In addition, the adsorption capacity of non- 

factor (IF) defines the adsorption capacity ratio of imprinted and non-imprinted aerogels and shows the separation capacity of the aerogels. When $\mathrm{pH}=5.0$, the maximum value is 1.420 , and the imprinted aerogel can achieve the highest adsorption capacity. Therefore, in subsequent adsorption experiments, we set the $\mathrm{pH}=5.0$.

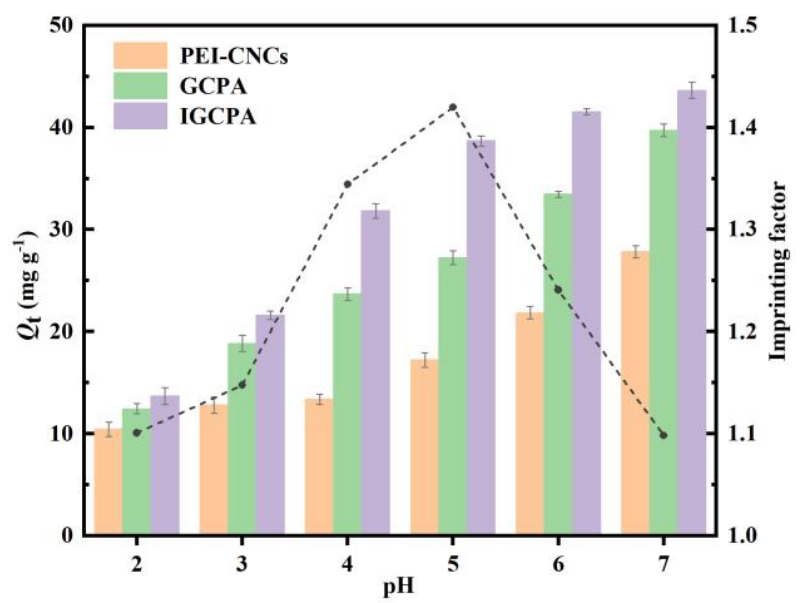

Figure 8 Effect of $\mathrm{pH}$ on the adsorption properties of PEI-CNCs, GCPA, IGCPA.

Adsorption dynamics: The relationship between adsorption quantity $\left(Q_{\mathrm{t}}\right)$ and contact time $(t)$ was discussed by adsorption dynamics experiments. As can be seen from Figure 9, the adsorption curve of aerogels grew rapidly at the beginning and reached $80 \%$ of the maximum adsorption capacity within 200 minutes. Then, the adsorption curve grew slowly and finally reached adsorption equilibrium within 6 hours, at which time the blot sites on the aerogels became saturated. The adsorption capacity adsorption rate constant and adsorption mechanism. The relevant calculation parameters of the adsorption kinetics model are shown in Table 1. Obviously, the PSOKM model $\left(R^{2} \geq 0.990\right)$ and the kinetic data fit well, indicating that the main 
adsorption process is chemical adsorption.

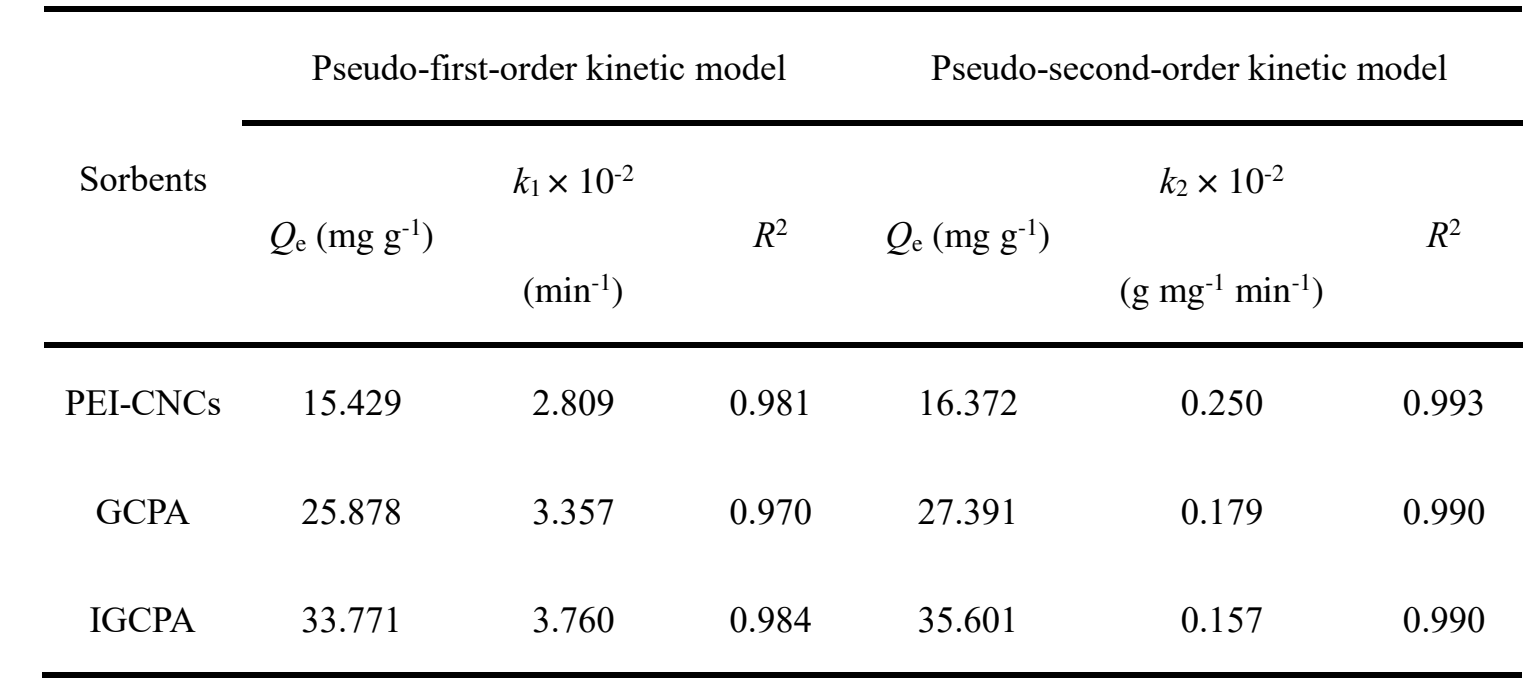

Figure 9 Kinetic data and modeling for the adsorption of Dy(III).

Table 1 Kinetic constants for the Pseudo-first-order and Pseudo-second-order models.

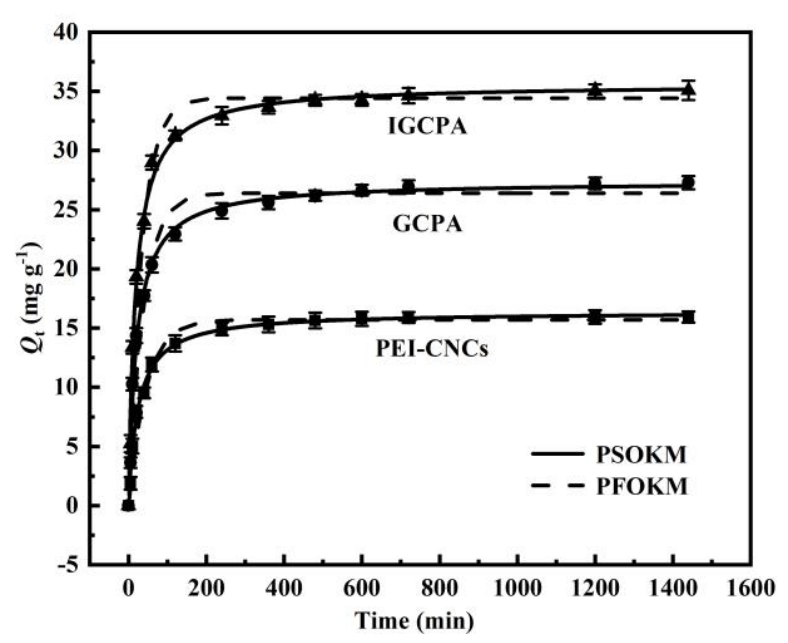


343 Its high adsorption capacity was attributed to the functionalization of CNCs by GO and

344 PEI, which provided more binding sites for Dy(III). Table 2 summarizes the relevant

345 isothermal constants. The larger $R^{2}$ value (0.991-0.994) indicated that the Langmuir

346 isothermal adsorption model could fit the experimental data well, and the adsorption

347 process by aerogels proved to be monolayer adsorption. The lower value of $1 / n$

348 indicates that IGCPA has better adsorption conditions for Dy(III) than GCPA.

349

350

351

352

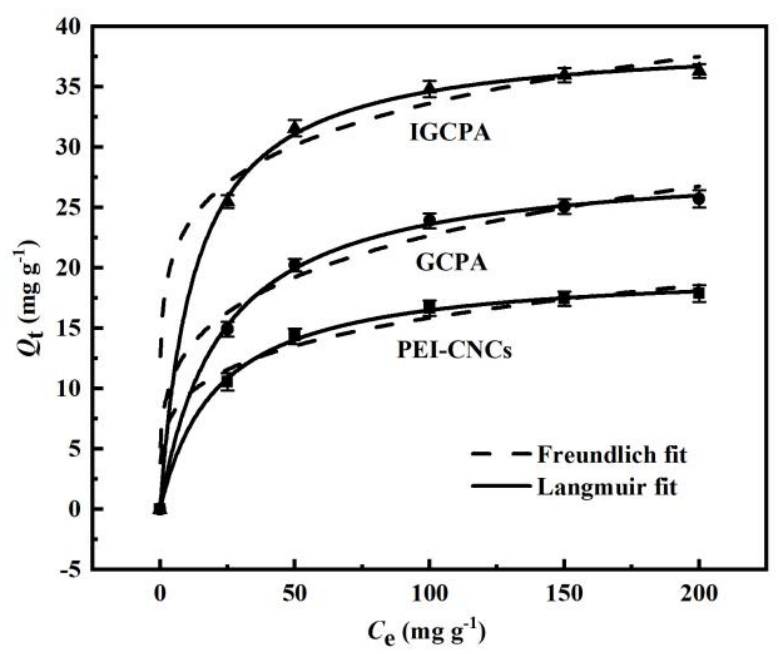

Figure 10 Adsorption isotherm of Dy(III) by aerogels.

Table 2 Adsorption equilibrium constants for Langmuir and Freundlich isotherm equations.

\begin{tabular}{ccccccc}
\hline & \multicolumn{3}{c}{ Langmuir } & \multicolumn{3}{c}{ Freundlich } \\
\cline { 2 - 7 } Sorbents & & $K_{\mathrm{L}} \times 10^{-2}$ & & & \\
& $Q_{\mathrm{m}\left(\mathrm{mg} \mathrm{g}^{-1}\right)}$ & & $R^{2}$ & $K_{\mathrm{F}}\left(\mathrm{mg} \mathrm{g}^{-1}\right)$ & $1 / n$ & $R^{2}$ \\
& & $\left(\mathrm{~L} \mathrm{mg}^{-1}\right)$ & & & & \\
\hline PEI-CNCs & 19.950 & 4.795 & 0.991 & 5.561 & 0.228 & 0.898 \\
GCPA & 28.911 & 4.451 & 0.994 & 7.574 & 0.238 & 0.909 \\
IGCPA & 39.027 & 7.838 & 0.991 & 16.306 & 0.157 & 0.881 \\
\hline
\end{tabular}

Adsorption thermodynamics: The effects of three kinds of aerogels on the 
$355 \Delta G^{\circ}$ was calculated according to the Gibb's free energy change equation (Figure 11), 356 and $\Delta H^{\circ}$ and $\Delta S^{\circ}$ are obtained by the relation between $\ln K^{\circ}$ and $1 / T$ (Figure 12). Table 3

357 lists three aerogels thermodynamics related parameters. $\Delta G^{\circ}$ is negative between -5.363 358 and $-6.742 \mathrm{~kJ} \mathrm{~mol}^{-1}$, indicating that the adsorption of Dy(III) is spontaneous, and the 359 higher the temperature, the more favorable the adsorption process. $\Delta H^{\circ}$ is positive, 360 indicating that the adsorption process is endothermic, which proves again that the 361 increase of temperature is conducive to adsorption. $\Delta S^{\circ}$ is positive, indicating that the 362 adsorption process is an entropy increase process. In summary, the adsorption of Dy(III) by aerogels is spontaneous, endothermic and entropy-enhancing.
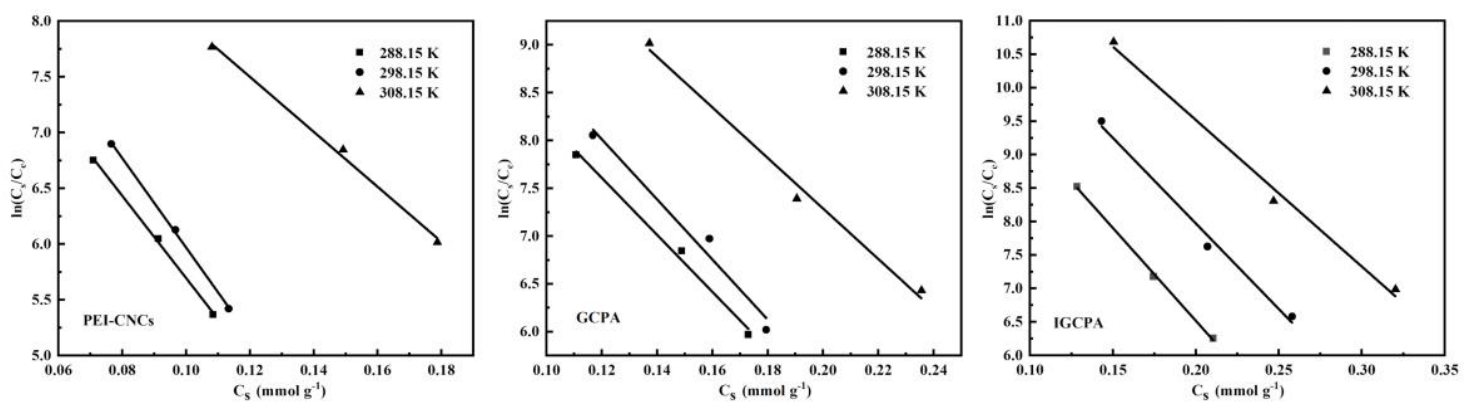

Figure 11 Thermodynamic properties of three aerogels at different temperatures: a plot of $\ln \left(C_{\mathrm{s}} / C_{\mathrm{e}}\right)$.

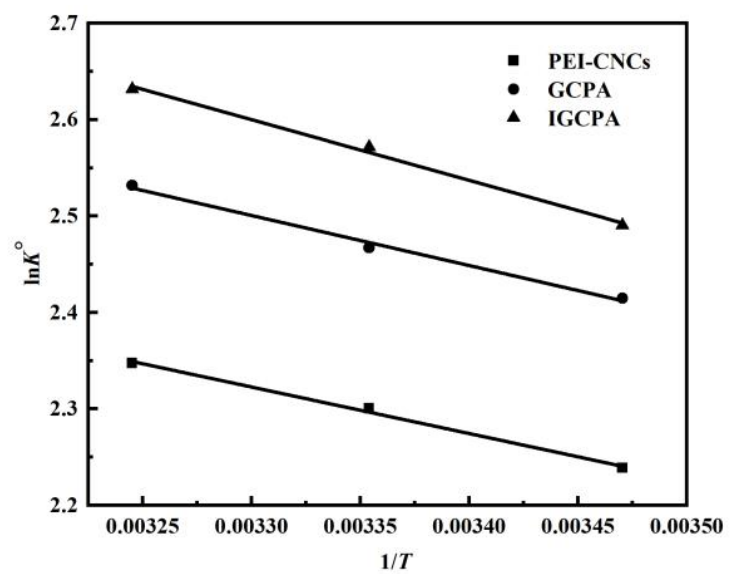

368 Figure 12 Thermodynamic properties of three aerogels at different temperatures: $\ln K^{\circ}$ 
Table 3 Thermodynamic parameters of three aerogels.

\begin{tabular}{|c|c|c|c|c|c|c|}
\hline Sorbents & $\Delta H^{\circ}\left(\mathrm{kJ} \mathrm{mol}^{-1}\right)$ & $\Delta S^{\circ}\left(\mathrm{J} \mathrm{mol}^{-1}\right)$ & $T(\mathrm{~K})$ & $K^{\circ}$ & $\Delta G^{\circ}\left(\mathrm{kJ} \mathrm{mol}^{-1}\right)$ & $R^{2}$ \\
\hline \multirow{3}{*}{ PEI-CNCs } & \multirow{3}{*}{4.011} & \multirow{3}{*}{32.547} & 288.15 & 9.382 & -5.363 & \multirow{3}{*}{0.993} \\
\hline & & & 298.15 & 9.979 & -5.702 & \\
\hline & & & 308.15 & 10.457 & -6.014 & \\
\hline \multirow{3}{*}{ GCPA } & \multirow{3}{*}{4.313} & \multirow{3}{*}{35.023} & 288.15 & 11.188 & -5.785 & \multirow{3}{*}{0.990} \\
\hline & & & 298.15 & 11.789 & -6.116 & \\
\hline & & & 308.15 & 12.577 & -6.487 & \\
\hline \multirow{3}{*}{ IGCPA } & \multirow{3}{*}{5.228} & \multirow{3}{*}{38.869} & 288.15 & 12.064 & -5.966 & \multirow{3}{*}{0.991} \\
\hline & & & 298.15 & 13.086 & -6.374 & \\
\hline & & & 308.15 & 13.897 & -6.742 & \\
\hline
\end{tabular}

Selective tests: In order to prove that IGCPA has good adsorption selectivity, a

372 competitive adsorption experiment was carried out. The aerogels adsorbed Dy(III) as

373 well as $\operatorname{Pr}(\mathrm{III})$ and $\mathrm{Nd}(\mathrm{III})$. The result is shown in Figure 13. The $K_{\mathrm{d}}$ value of IGCPA

374 for Dy(III) is higher than that of GCPA, the maximum $K_{\mathrm{d}}$ value is $1909.2 \mathrm{~mL} \mathrm{~g}^{-1}$ of

375 IGCPA. It can be seen that the unique imprinted hole on IGCPA has a specific

376 adsorption on Dy(III), and this site cannot adsorb other rare earth ions. Compared with

377 other adsorption experiments, the adsorption capacity of IGCPA for Dy(III) in this

378 experiment decreased, indicating that other rare earth ions would interfere with the

379 adsorption of Dy(III). Therefore, IGCPA can preferentially separate Dy(III) from the 380 multi-ion coexisting system. 


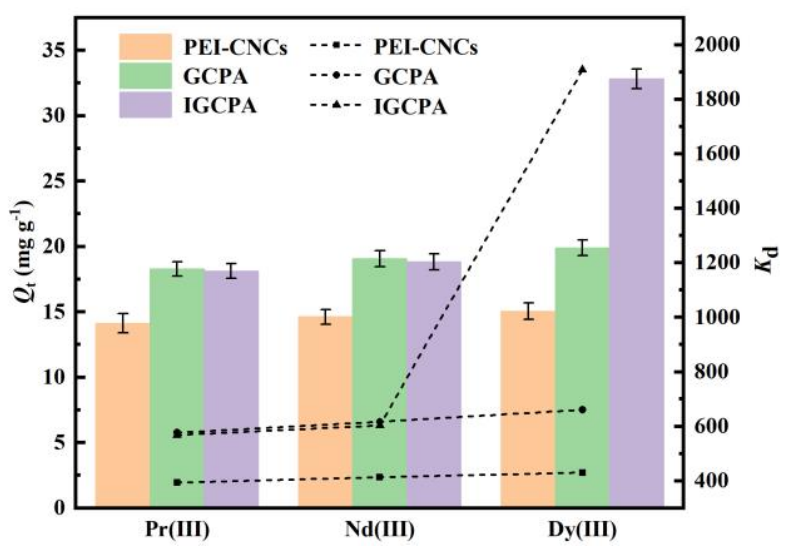

Figure 13 The adsorption capacity of PEI-CNCs, GCPA and IGCPA for various ions and their $K_{\mathrm{d}}$ values.

Regeneration: The reuse of adsorbents will minimize industrial production costs.

As can be seen from Figure 14, five adsorption cycles were performed to test the reliability of the aerogel. After 5 cycles, the adsorption capacity decreased to 67.97$79.75 \%$ of the initial adsorption capacity, which was mainly due to the loss of adsorption sites caused by eluent elution. In conclusion, IGCPA has reliable repeatability and is expected to be a commercially available adsorption material.

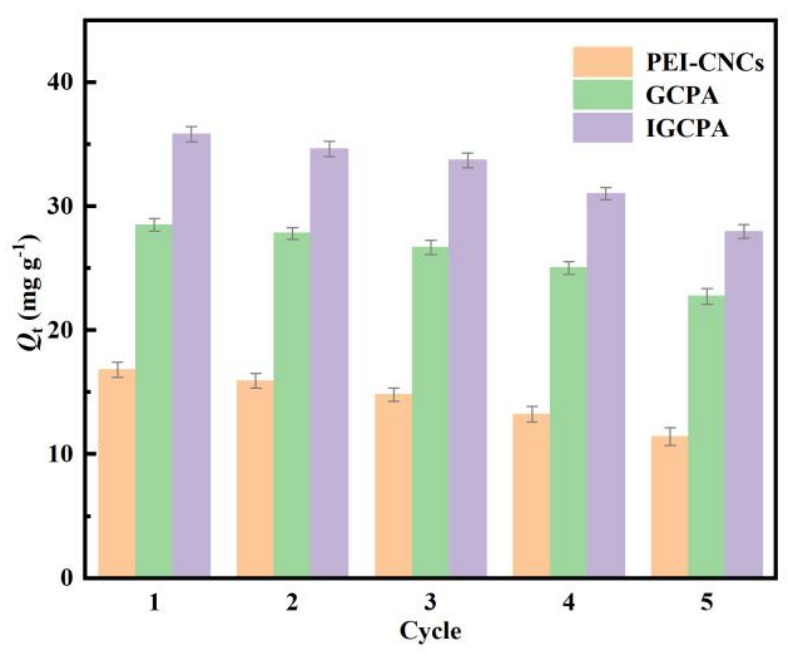

Figure 14 Regeneration of aerogels over 5 cycles.

\section{Conclusions}

In this study, under the circumstance of environmental pollution caused by rare earth elements and urgent need to reduce the cost, the imprinted aerogel IGCPA with 
periodic structure was prepared from biodegradable cellulose nanocrystals and used for the specific adsorption of Dy(III) in rare earth waste. GO and PEI were uniformly distributed on the CNCs skeleton and did not affect the crystallization zone. The carboxyl group introduced by surface modification further improved the adsorption capacity of Dy(III) as synergistic functional monomer. The results showed that the optimum $\mathrm{pH}$ value for adsorption was 5.0. PSOKM can fit the adsorption data well, indicating that aerogel adsorption of Dy(III) is mainly a chemical process. According to the adsorption isotherm, the adsorption process was mainly monolayer adsorption, and the maximum adsorption capacity was $39.027 \mathrm{mg} \mathrm{g}^{-1}$. In multi-ion coexisting systems, imprinted aerogels tend to adsorb Dy(III). In addition, the repeated use test shows that the material has strong regeneration performance. The results show that the composite imprinted aerogel can adsorb Dy(III) efficiently and greenly.

\section{Acknowledgement}

The authors thank the National Natural Science Foundation of China (Grant Nos. 21876015, 21808018, 21878026, 22008014), Postgraduate Research \& Practice Innovation Program of Jiangsu Province (No. KYCX20_2579, No. KYCX20_2592 and No. KYCX20_2595). Also, the author would like to thank the researchers at the Analytical Testing Center of Changzhou University for their assistance in SEM, FTIR and TG analysis.

\section{Declarations}

\section{Conflict of interest}

The authors declare that they have no conflict of interest.

\section{Ethical approval}

The article does not contain any experiments with human participants or animals 
performed by any of the authors.

\section{Informed consent}

Informed consent was obtained from all individual participants included in the study.

\section{References}

Alcaraz L, Escudero ME, Alguacil FJ, Llorente I, Urbieta A, Fernández P, López FA (2019) Dysprosium Removal from Water Using Active Carbons Obtained from Spent Coffee Ground Nanomaterials 9 doi:10.3390/nano9101372

Almeida APC, Canejo JP, Fernandes SN, Echeverria C, Almeida PL, Godinho MH (2018) Cellulose-Based Biomimetics and Their Applications Advanced Materials 30 doi:10.1002/adma.201703655

Ashour RM et al. (2017) Rare Earth Ions Adsorption onto Graphene Oxide Nanosheets Solvent Extraction and Ion Exchange 35:91-103 doi:10.1080/07366299.2017.1287509

Balaram V (2019) Rare earth elements: A review of applications, occurrence, exploration, analysis, recycling, and environmental impact Geoscience Frontiers 4: 1285-1303 doi:10.1016/j.gsf.2018.12.005

Bisaka K, Thobadi IC, Pawlik C (2017) Extraction of rare earths from iron-rich rare earth deposits J S Afr Inst Min Metall 117:731-739 doi:10.17159/24119717/2017/v117n8a2

Brinkmann A, Chen M, Couillard M, Jakubek ZJ, Leng T, Johnston LJ (2016) 

32:6105-6114 doi:10.1021/acs.langmuir.6b01376

Cao Y, Wang PX, D'Acierno F, Hamad WY, Michal CA, MacLachlan MJ (2020) Tunable Diffraction Gratings from Biosourced Lyotropic Liquid Crystals Advanced Materials 32 doi:10.1002/adma.201907376

Du A, Zhou B, Zhang Z, Shen J (2013) A Special Material or a New State of Matter: A Review and Reconsideration of the Aerogel Materials 6:941-968 doi:10.3390/ma6030941

Du H, Liu W, Zhang M, Si C, Zhang X, Li B (2019) Cellulose nanocrystals and cellulose nanofibrils based hydrogels for biomedical applications Carbohydrate Polymers 209:130-144 doi:10.1016/j.carbpol.2019.01.020

Fang L, Miao Y, Wei D, Zhang Y, Zhou Y (2021) Efficient removal of norfloxacin in water using magnetic molecularly imprinted polymer Chemosphere 262:128032 doi:10.1016/j.chemosphere.2020.128032

Fu J, Chen L, Li J, Zhang Z (2015) Current status and challenges of ion imprinting Journal of Materials Chemistry A 3:13598-13627 doi:10.1039/c5ta02421h

Fujiwara I, Takeuchi K, Murakami Y (2016) Development of a Dysprosium (III) Ionselective Electrode Using a Dysprosium (III)-imprinted Polymer Bunseki Kagaku 65:527-531 doi:10.2116/bunsekikagaku.65.527

George J, S N S (2015) Cellulose nanocrystals: synthesis, functional properties, and applications Nanotechnology, Science and Applications doi:10.2147/nsa.S64386 
Habib K (2019) A products classification approach to optimize circularity of critical resources-the case of NdFeB magnets Journal of Cleaner Production:90-97 doi:10.1016/j.jclepro.2019.05.048

Huang H, Zhu JJ (2019) The electrochemical applications of rare earth-based nanomaterials Analyst 144:6789-6811 doi:10.1039/c9an01562k

Jiang W, Shen P, Yi J, Li L, Wu C, Gu J (2020) Surface modification of nanocrystalline cellulose and its application in natural rubber composites Journal of Applied Polymer Science 137 doi:10.1002/app.49163

Kaneko T, Hikosaka R, Nagata F, Inagaki M, Kato K (2019) Effective adsorption of dysprosium ions on amino and carboxyl functionalized mesoporous silica sheets J Asian Ceram Soc 7:213-220 doi:10.1080/21870764.2019.1606139

Kegl T, Ban I, Lobnik A, Košak A (2019) Synthesis and characterization of novel $\gamma$ Fe2O3-NH4OH@SiO2(APTMS) nanoparticles for dysprosium adsorption Journal of Hazardous Materials 378 doi:10.1016/j.jhazmat.2019.120764

Kim J, Sadasivuni KK, Zhai L, Gao X, Jo EB (2014) Cellulose Nanocrystals and Nanofibers for Smart Optics Materials. Paper presented at the 2014 International Symposium on Optomechatronic Technologies,

Kontturi E, Laaksonen P, Linder MB, Nonappa, Groschel AH, Rojas OJ, Ikkala O (2018) Advanced Materials through Assembly of Nanocelluloses Adv Mater 30:e1703779 doi:10.1002/adma.201703779

Liang XD, Ye M, Yang L, Fu WB, Li Z (2018) Evaluation and Policy Research on the Sustainable Development of China's Rare Earth Resources Sustainability 10:16 
Long L-Y, Weng Y-X, Wang Y-Z (2018) Cellulose Aerogels: Synthesis, Applications, and Prospects Polymers 10 doi:10.3390/polym10060623

Mahdi Z, Yu QJ, El Hanandeh A (2018) Competitive adsorption of heavy metal ions $(\mathrm{Pb} 2+, \mathrm{Cu} 2+$, and $\mathrm{Ni2}+)$ onto date seed biochar: batch and fixed bed experiments Separation Science and Technology 54:888-901 doi:10.1080/01496395.2018.1523192

Ni'am AC, Wang Y-F, Chen S-W, Chang G-M, You S-J (2020) Simultaneous recovery of rare earth elements from waste permanent magnets (WPMs) leach liquor by solvent extraction and hollow fiber supported liquid membrane Chemical Engineering and Processing - Process Intensification 148 doi:10.1016/j.cep.2020.107831

Peng Y, Gardner DJ, Han Y (2011) Drying cellulose nanofibrils: in search of a suitable method Cellulose 19:91-102 doi:10.1007/s10570-011-9630-z

Prodius D, Gandha K, Mudring AV, Nlebedim IC (2020) Sustainable Urban Mining of Critical Elements from Magnet and Electronic Wastes ACS Sustain Chem Eng 8:1455-1463 doi:10.1021/acssuschemeng.9b05741

Salimian S, Zadhoush A, Naeimirad M, Kotek R, Ramakrishna S (2018) A review on aerogel: 3D nanoporous structured fillers in polymer-based nanocomposites Polym Compos 39:3383-3408 doi:10.1002/pc.24412

Sato K, Mochizuki H, Okajima K, Yamane C (2004) Effects of Hydrophobic Solvents on X-Ray Diffraction Patterns of Regenerated Cellulose Membrane Polymer 
Stepanova M, Averianov I, Gofman I, Solomakha O, Nashchekina Y, Korzhikov-Vlakh

Zhang J, Zhang J (2020) Advanced functional materials based on cellulose Acta Polymerica sinica:1376-1398

Zhang L, Li L, Shi D, Peng X, Song F, Nie F (2019) Kinetics and mechanism study of lithium extraction from alkaline solution by HFTA and TOPO and stripping process using Lewis cell technique Sep Purif Technol 211:917-924 doi:10.1016/j.seppur.2018.10.043 
Zhang Y, Bian T, Jiang R, Zhang Y, Zheng X, Li Z (2021) Bionic chitosan-carbon imprinted aerogel for high selective recovery of $\mathrm{Gd}()$ from end-of-life rare earth productions J Hazard Mater 407:124347 doi:10.1016/j.jhazmat.2020.124347

Zhao F et al. (2017) Polyethylenimine-cross-linked cellulose nanocrystals for highly efficient recovery of rare earth elements from water and a mechanism study Green Chemistry 19:4816-4828 doi:10.1039/c7gc01770g

Zhao H, Xia J, Yin D, Luo M, Yan C, Du Y (2019) Rare earth incorporated electrode materials for advanced energy storage Coordination Chemistry Reviews 390:32-49 doi:10.1016/j.ccr.2019.03.011

Zheng XD, Zhang Y, Bian TT, Zhang YZ, Li ZY, Pan JM (2020) Oxidized carbon materials cooperative construct ionic imprinted cellulose nanocrystals films for efficient adsorption of Dy(III) Chem Eng J 381:10 doi:10.1016/j.cej.2019.122669

Zhu Y, Niu Y, Li H, Ren B, Qu R, Chen H, Zhang Y (2018) Removal of Cd(II) and Fe(III) from DMSO by silica gel supported PAMAM dendrimers: Equilibrium, thermodynamics, kinetics and mechanism Ecotoxicol Environ Saf 162:253-260 doi:10.1016/j.ecoenv.2018.06.094 